\title{
COMPARACIÓN DE LA RESISTENCIA AL DESALOJO DE POSTES PREFABRICADOS EN DIENTES UNIRADICULARES: UN ESTUDIO IN VITRO
}

\author{
${ }^{1}$ Juliana Elisa Celis Corzo, ${ }^{1}$ Adriana Cáceres Marulanda, ${ }^{2}$ Juan Carlos Cabrera Rojas, \\ ${ }^{3}$ Jorge Guillermo Díaz Rodríguez \\ ${ }^{1}$ Estudiante X Semestre F. de Odontología U. Santo Tomás, Colombia. ${ }^{2}$ Odontólogo U. Santo Tomás, Especialista en Rehabilitación Oral, \\ U. Santo Tomás, Docente U. Santo Tomás, Colombia. \\ ${ }^{3}$ Ingeniero Mecánico U. Industrial de Santander, Master of Science Mechanical Engineering U. of North Texas, Docente U. Santo Tomás, Colombia.
}

Autor responsable de correspondencia: Juan Carlos Cabrera Rojas

Correo electrónico: juanc_1970@yahoo.com.mx

RESUMEN

Objetivo: comparar dos técnicas de cementación de postes en fibra de vidrio en dientes con paredes radiculares debilitadas.

Materiales y métodos: se realizó un estudio experimental In vitro. Se recolectaron 60 piezas dentales y se dividieron en tres grupos de manera aleatoria (A, B y C), se empleó la técnica convencional con cemento resinoso en 20 dientes con conductos angostos y en 20 dientes con paredes radiculares debilitados (grupos A y B). La técnica de refuerzo radicular con resina fluida y cemento resinoso se uso en los 20 dientes restantes (grupo C) que presentaban paredes radiculares debilitadas. Se evaluó la fuerza de desalojo a la tracción en los tres grupos mediante una Máquina Universal de Ensayos para medir la fuerza de desalojo a la tracción. Se utilizó el tes de Mann Whitney y el ANOVA de una vía con un valor de $\mathrm{p}<0,05$ como estadísticamente significativo.

Resultados: no se encontró una diferencia estadísticamente significativa al comparar la resistencia al desalojo en los tres grupos $(\mathrm{p}=0,064)$ pero si se observó al analizar las fuerzas de tracción $(\mathrm{p}=0,005)$. Además, se evidenció que existe menor adhesión entre la interfase dentina-material restaurador en los tres grupos y que el comportamiento presentado entre poste-cemento indicó que los postes presentaron mayor adhesión con el cemento resinoso.

Conclusión: el grupo C presentó mayor resistencia a la fuerza de tracción posiblemente, debido a que la resistencia de la estructura dental se incrementó cuando el canal radicular fue reforzado con resina compuesta. La técnica convencional fue efectiva en el caso de rehabilitar dientes con conductos radiculares angostos. [Celis JE, Cáceres A, Cabrera JC, Díaz JG. Comparación de la resistencia al desalojo de postes prefabricados en dientes uniradiculares: un estudio In vitro. Ustasalud 2013; $12: 55$ - 62]

Palabras clave: Resistencia a la tracción, Dentina, Cemento dental.

\section{COMPARISON OF RESISTANCE IN PRECAST POST EVICTION UNIRADICULAR TEETH: AN IN VITRO STUDY}

\begin{abstract}
Objective: To compare two cementing techniques in uniradicular teeth with root weakened walls.

Methods: An in vitro experimental study was done with sixty collected teeth which were divided into three groups at random (A, B and C). The conventional technique with resin cement was done in 20 teeth with narrow canals 20 other teeth with weakened root walls (groups A and B). The technique of root reinforcement and flowable resin cement was used in the remaining 20 teeth (group C) whch had weakened root walls. The dislodging force in the three groups was tested using an universal testing machine. The Mann Whitney and one-way ANOVA tests were used with a p value $<0.05$ as statistically significant.

Results: It was not found a statistically significant difference when comparing the dislodging force in the three groups ( $\mathrm{p}=0.064$ ) but, it was found a difference when comparing traction forces $(\mathrm{p}=0.005)$. In addition, it was observed that there was less adhesion between dentin - restorative interface materials in the three groups. The behavior exhibited between said post - cement poles showed higher adhesion with the resin cement.

Conclusion: Group C showed greater resistance to the tensile force perhaps because the resistance of the tooth structure was increased when the root canal was reinforced with composite resine. The conventional technique was effective in the case of restoring teeth with narrow root canals.
\end{abstract}

Keywords: Tensile strength, Dentin, Dental cementum

Recibido para publicación: mayo 30 de 2013. Aceptado para publicación: junio 20 de 2013. 


\section{INTRODUCCIÓN}

Rehabilitar dientes tratados endodónticamente merece una atención especial puesto que por lo general, deben ser sometidos a largos procedimientos clínicos para garantizar su durabilidad en boca. Las causas más frecuentes que indican la necesidad de conservar dientes con importantes pérdidas de tejido remanente son: la fractura coronal, las caries dentales extensas, los traumas y las maniobras inadecuadas que se realizan cuando se desobturan los conductos. Todo esto conlleva a buscar la mejor manera de brindar un refuerzo a aquellas paredes radiculares que han quedado susceptibles a la fractura.

La búsqueda de la restauración ideal para dientes tratados endodónticamente ha sido muy compleja debido a las variaciones anatómicas, extensión de la destrucción, posición en la boca, cantidad de hueso remanente y la función designada ya sea como restauración individual o soporte de prótesis fija; ${ }^{1}$ todo esto ha complicado la selección del tipo de tratamiento para cada situación específica. Aún no se ha logrado identificar la técnica adhesiva ideal para rehabilitar dientes con paredes radiculares debilitadas, a pesar del esfuerzo de investigadores y todos los adelantos tecnológicos. Se requiere de un método que sea perdurable en el tiempo, de fácil ejecución y aplicable en forma general, ya que hay muchos factores involucrados en el éxito, entre los cuales se encuentran, los mismos materiales, el sustrato dental y el factor humano que lo ejecuta. ${ }^{1}$

En condiciones normales la dentina provee una base sólida para la restauración de dientes. El esfuerzo que soporta depende de su cantidad así como su integridad y forma anatómica. ${ }^{2}$ Sin embargo, posterior al tratamiento endodóntico hay una pérdida considerable de dentina, por lo que es fundamental la cantidad de tejido remanente sano para retener la corona definitiva. ${ }^{3}$ Debido a esto, en los dientes tratados endodónticamente aumentan las posibilidades de fractura. Se ha recomendado reforzar los conductos debilitados con resina y así poder restaurarlos con un anclaje radicular para establecer la retención necesaria para la restauración posterior. Existen diferentes alternativas y materiales para cementar postes en fibra de vidrio en dientes con paredes radiculares debilitadas. ${ }^{4}$ Fakiha y colaboradores establecieron que los cementos resinosos adhesivos ofrecen mayor capacidad retentiva al compararlos con los de fosfato de zinc y de ionómero de vidrio. ${ }^{5}$ Ojeda y colaboradores concluyen que "los endopostes vaciados fracturaron todos los dientes cuando recibieron fuerza de compresión, actuando como cuña. Los endo- postes vaciados soportaron mejor las fuerzas de tensión o desalojo debido a que es una sola estructura y presenta una superficie mayor de contacto entre la superficie interna de la preparación y el poste". ${ }^{6}$

Dada la alta frecuencia de conductos radiculares debilitados por tratamientos endodonticos o caries extensas, el diámetro de la dentina radicular que permanece es menor al que la literatura ha reportado como predecible para evitar fracturas. El mínimo de dentina remanente indicado para restaurar con postes y realizar un refuerzo radicular debe ser de $0,5 \mathrm{~mm} .{ }^{6}$ Por esto es evidente la necesidad de estudiar y proponer métodos o técnicas de cementación de postes eficaces en conductos altamente destruidos que permita utilizar un anclaje para retener la restauración final y así devolverle la funcionalidad al diente.

Además, los procedimientos de cementación son de suma importancia ya que existen cambios drásticos que repercuten en la práctica diaria, como lo es el desuso de la técnica de grabado total, así como el auge de los agentes cementantes autoadhesivos en la técnica de adhesión. Los cementos resinosos son materiales utilizados para la cementación y poseen composición semejante a la de las resinas compuestas restauradoras. ${ }^{7}$ Esto es una matriz orgánica formada por Bis-GMA (bisfenol glicidil metacrilato) o UEDMA (uretano dimetacrilato) y monómeros de bajo peso molecular, como el TEGDMA (trietilenoglicol dimetacrilato). Estos poseen también agrupamientos funcionales hidrofilicos para promover la adhesión a la dentina como el HEMA (hidroxietil metacrilato), el 4-META (4-metacriloxietil trimelitano anidro) y el MDP (10-metacriloxidecil dihidrógeno fosfato). ${ }^{8}$ Por su numerosa variedad de colores y alta resistencia, los cementos de resina se indican para cementar coronas total-cerámica, "inlays y onlays" y carillas de cerámica. ${ }^{9}$

Garita y Rodríguez sugirieron que las funciones del material que se utiliza como refuerzo son fundamentalmente mecánicas, resistentes para permitir comportamientos anisotrópicos. ${ }^{10}$ Por su parte, García realizó un estudio que evaluó la relación entre la resistencia a la tensión del cemento de ionómero vítreo y su resistencia adhesiva a los metales, mostró una relación muy estrecha entre las fuerzas de tensión y las de adhesión, con lo cual concluyó que el cemento de ionómero vítreo al resistir altas fuerzas mecánicas es muy bueno para la adhesión en superficies metálicas. ${ }^{11}$

Así mismo, Saupe y colaboradores concluyeron que los postes deben resistir las fuerzas externas que pretenden desalojarlos del lecho generado en la raíz dentaria. ${ }^{12}$ Las tensiones máximas soportadas por 
el poste antes de su desprendimiento constituyen su resistencia al desalojo. ${ }^{13}$ Por lo tanto, el objetivo de este trabajo fue comparar dos técnicas de cementación en dientes con paredes radiculares debilitadas e identificar la interface donde ocurre el modo de falla.

\section{MATERIALES Y MÉTODOS}

Se realizó un estudio experimental In vitro. Con base en los estudios realizados por Mazitelli y Rodríguez, ${ }^{14,15}$ se incluyeron 60 dientes con las siguientes características: dientes incisivos centrales superiores, caninos superiores e inferiores, sin daño en la estructura radicular y que no presentaban dilaceraciones. Se excluyeron dientes con fractura radicular, con conductos radiculares calcificados y dientes con caries radicular.

Los dientes fueron conservados en suero fisiológico hasta el momento de ser utilizados. Se obtuvieron radiografias en dirección proximal de cada pieza dental para demostrar que cumplían con los criterios de inclusión y se removió la corona de cada uno de los dientes, con un disco de carburo para dejar todas las raíces a $16 \mathrm{~mm}$ de longitud. Para el tratamiento de endodoncia fue utilizada la técnica de instrumentación Stepback, con limas tipo-K de acero inoxidable (Dentsply/Maillefer ${ }^{\circledR}$ ). Al terminar de la instrumentación, se irrigó con hipoclorito de sodio $(\mathrm{NaClO})$ al $5,25 \%$ para obturar con la técnica de condensación lateral y vertical empleando conos de gutapercha y cemento Grossman.

Seguidamente se hizo la desobturación con el fin de alojar el poste, se retiró la gutapercha por medios mecánicos (fresas Gates y Glidden No. 1 y 2 para conductos estrechos y No. 3, 4, 5 y 6 para grupos con conductos amplios). Nuevamente, se irrigó con hipoclorito para eliminar el smear layer, se limpiaron los conductos con agua y aire a presión y se secaron con conos de papel. Los dientes se colocaron en cubos de resina acrílica, con el fin de ser ajustados a las mordazas de la Máquina Universal de Ensayos Shimadzu Autograph AG-i 250 con celda de carga de $1 \mathrm{kN}$ (Figura 1).

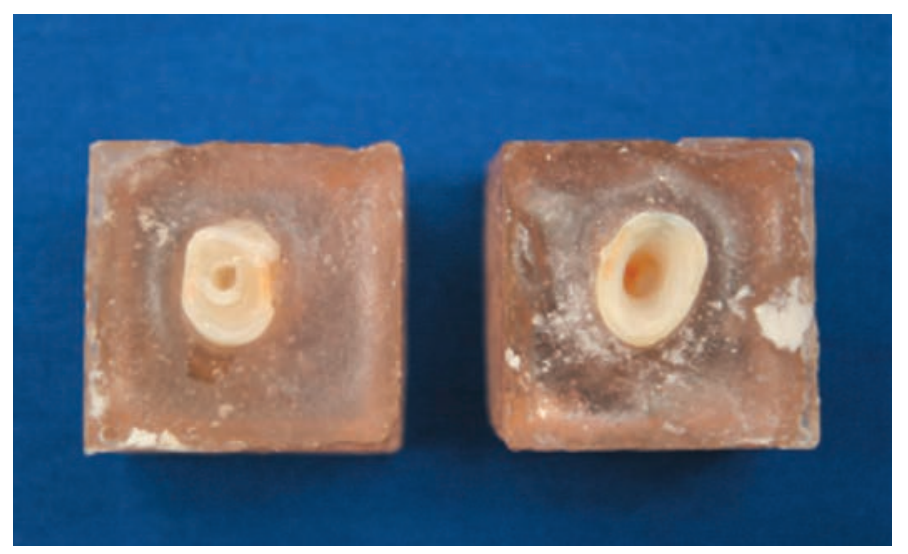

Figura 1. Muestras colocadas en cubos de resina acrílica.

Por último, se procedió a dividir al azar las 60 muestras en tres grupos (A, B y C) de tal manera que quedaron 20 dientes en cada grupo. Las diferentes técnicas de cementación de los postes se distribuyeron de la siguiente manera:

Grupo A: se restauraron 20 dientes con técnica convencional en conductos angostos, se cementaron los postes en fibra de vidrio con un cemento resinoso (Relyx U200, 3M ESPE ${ }^{\circledR}$ ). La Figura 2 muestra el proceso para este grupo.

Grupo B: se restauraron 20 dientes con la técnica convencional en conductos amplios (paredes radiculares debilitadas) al cementar los postes en fibra de vidrio con un cemento resinoso (Relyx U200, 3M ESPE ${ }^{\circledR}$ ). La Figura 3 muestra el proceso para este grupo.

Grupo C: se restauraron 20 dientes con la técnica del refuerzo radicular en conductos amplios (pare- des radiculares debilitadas), se cementaron postes en fibra de vidrio con un refuerzo en resina fluida y cemento resinoso; el poste se envolvió con cinta aislante para que guardara el espacio del cemento (Figura 4).

Todas las muestras fueron sometidas a fuerzas de tracción vertical usando la Máquina Universal de Ensayos mencionada como se muestra en la Figura 5. La fuerza reportada se hace al registrar un desalojo de de $1 \mathrm{~mm}$ en los postes. Los datos fueron registrados y graficados por el software Trapezium 2,23. Los datos fueron registrados en una base de datos en Excel creada para tal fin. Posteriormente, se exportaron al programa estadístico SPSS. Para el análisis estadístico, se obtuvieron medidas de tendencia central y dispersión; se utilizó el test de Mann Whitney y el Análisis de Varianza (ANOVA) de una vía. 


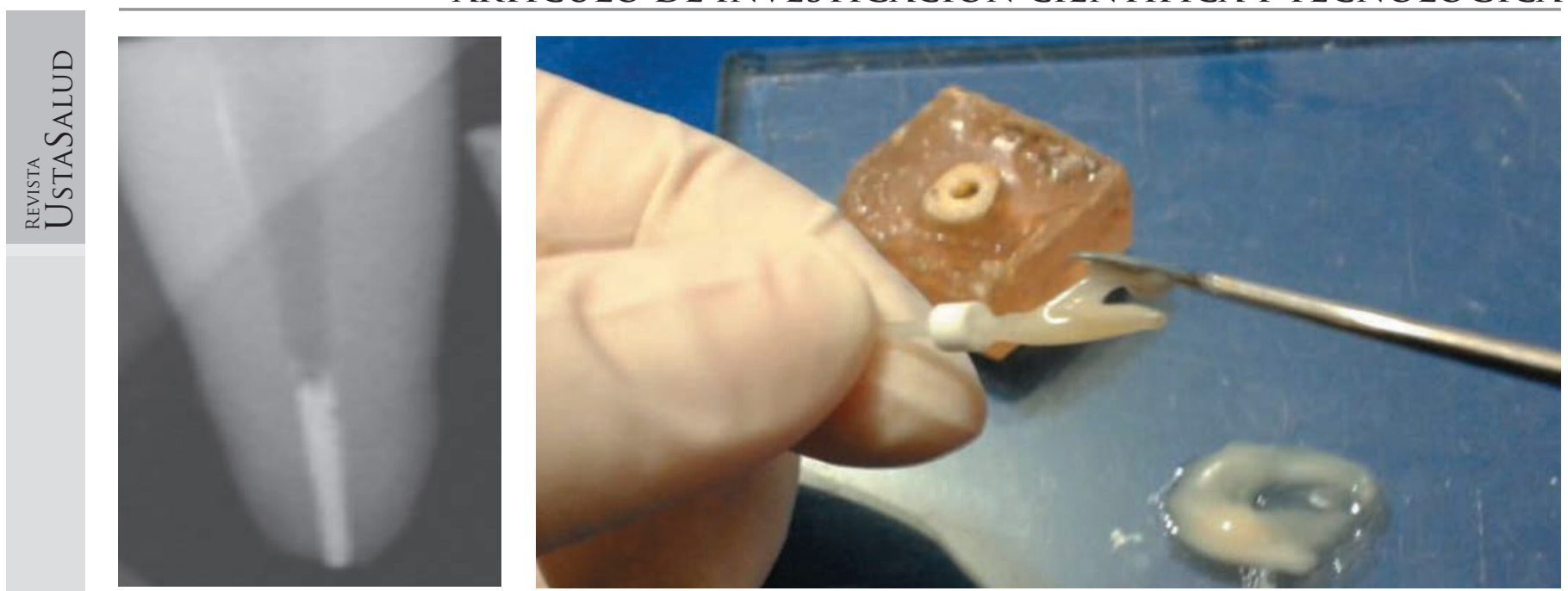

Figura 2. Restauración grupo A con conductos angostos.


Figura 3. Restauración grupo con paredes radiculares debilitadas y cemento resinoso.
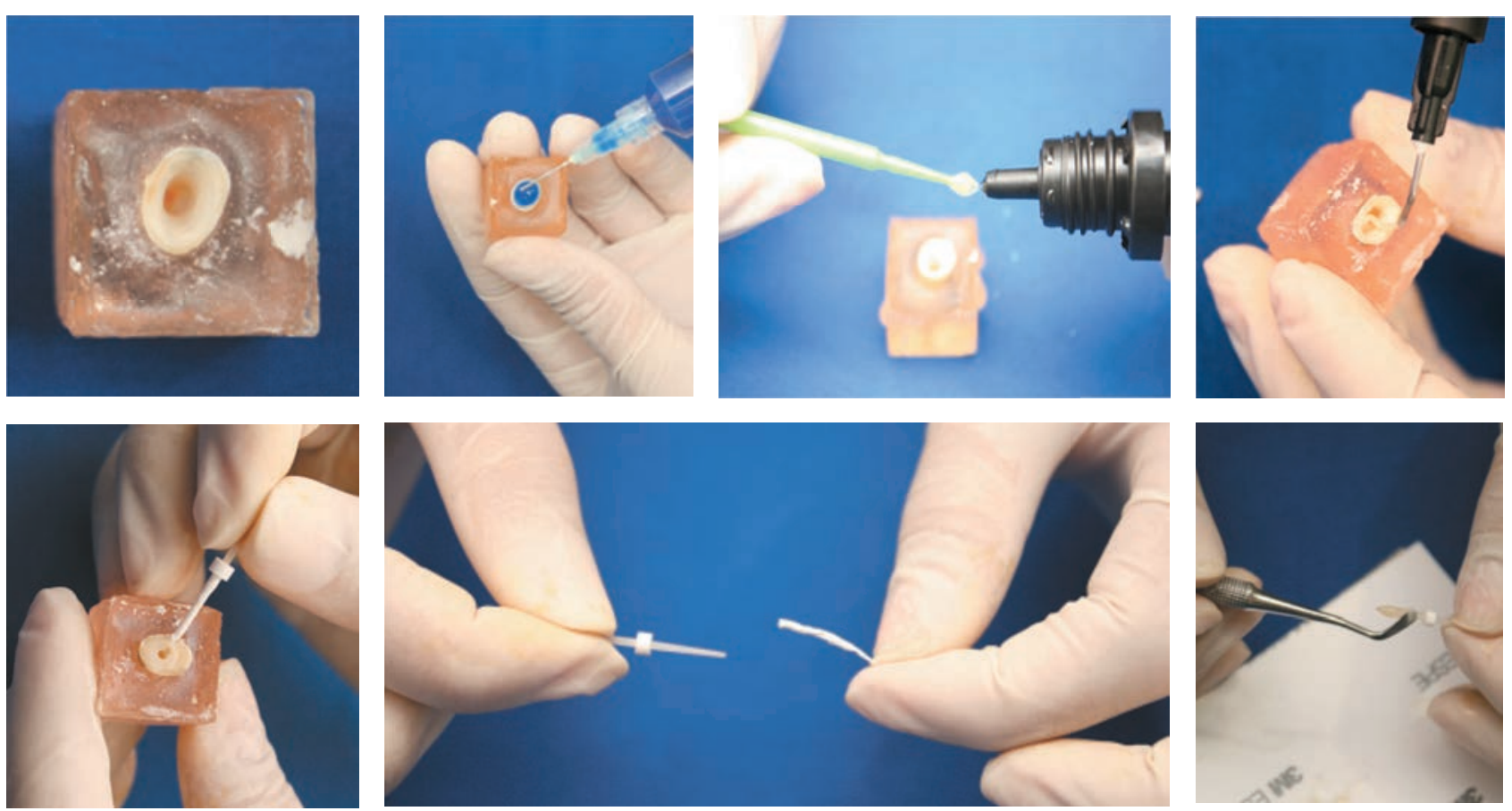

Figura 4. Restauración grupo con paredes radiculares debilitadas cemento resinoso y refuerzo en resina fluida. 



Figura 5. Montaje experimental en curso.

\section{RESULTADOS}

De los sesenta dientes incluidos en el estudio, cinco de ellas (2 del grupo A y 3 del grupo B) no proporcionaron medición alguna ya que se fracturaron los cubos 0 los postes. Los especímenes no fueron reemplazados puesto que de acuerdo con los estudios revisados el número mínimo de muestras era de diez. Como consecuencia de lo anterior, la muestra final se redujo a 55 dientes y los grupos procesados quedaron conformados de la siguiente manera: se probaron 18 dientes cementados con la técnica convencional y con conductos angostos como grupo control positivo, 17 dientes con la técnica de cementación convencional en dientes con conductos amplios y 20 dientes con la técnica de refuerzo radicular en conductos amplios. La técnica de cementación convencional correspondió al 60,3\% del total de las muestras, la cual se aplicó a los dientes de los grupos A y B como se observa en la Tabla 1.

Tabla 1. Análisis de las variables diente y técnica de cementación, frecuencias y porcentajes a nivel general

\begin{tabular}{lcc}
\hline \multicolumn{1}{c}{ Variable } & Categoría & n (\%) \\
\hline Tipo de diente & A & $18(31,0)$ \\
& B & $17(29,3)$ \\
Técnica de cementación & C & $20(34,5)$ \\
& 1 & $35(60,3)$ \\
\hline
\end{tabular}

En una descripción general de las variables cuantitativas (fuerza de tracción y resistencia al desalojo), se obtuvieron las medidas de tendencia central (promedio, mediana) y las medidas de dispersión (desviación estándar, valor mínimo y máximo). La mayor fuerza de tracción y resistencia al desalojo la presentó el grupo C, con un promedio de $212,8 \mathrm{~N}$ y $159,31 \mathrm{~N}$, respectivamente (Tabla 2). Aunque al grupo $\mathrm{C}$ se le aplicó la técnica de refuerzo radicular no se evidenció una diferencia estadísticamente significativa entre las técnicas de cementación.

Se realizó un análisis entre la resistencia al desalojo con las medidas del conducto en este caso angosto (grupo A) y amplios (grupo B y C), con el fin de encontrar una diferencia entre estas medidas. La prueba no paramétrica U. de Mann Withney indicó una diferencia estadísticamente significativa al obtener como resultado $\mathrm{p}=0,029$ al comparar la resistencia al desalojo con la medida del conducto (Tabla 3).

Adicionalmente, cuando se comparó la fuerza de tracción con las medidas del conducto se encontró una diferencia estadísticamente significativa $(p=0,003)$, es decir, la medida del conducto (angosto o amplio) influyó en la resistencia al desalojo según el grosor del conducto radicular. 
ARTÍCULO DE INVESTIGACIÓN CIENTÍFICA Y TECNOLÓGICA

Tabla 2. Variables cuantitativas fuerza de tracción y resistencia al desalojo

\begin{tabular}{lcccccc}
\hline \multicolumn{1}{c}{ Variable } & Grupo & Promedio & Mediana & $\begin{array}{c}\text { Desviación } \\
\text { estándar }\end{array}$ & Mínimo & Máximo \\
\hline \multirow{3}{*}{ Fuerza de tracción } & A & 103,3 & 63,5 & 48,3 & 25,0 & 199,0 \\
& B & 136,1 & 101,0 & 48,0 & 51,0 & 205,0 \\
\multirow{2}{*}{ Resistencia al desalojo } & C & 212,8 & 148,0 & 98,8 & 17,0 & 309,0 \\
& A & 79,2 & 43,0 & 50,7 & 0,4 & 186,9 \\
& B & 99,8 & 52,6 & 51,3 & 13,2 & 189,3 \\
\hline
\end{tabular}

Tabla 3. Comparación de la resistencia al desalojo con la medida del conducto

\begin{tabular}{|c|c|c|}
\hline Grupo & Mediana resistencia al desalojo & $\mathbf{P}^{\mathrm{a}}$ \\
\hline Angosto (A) & 43,0 & \\
\hline Amplio (B) & 52,6 & 0,029 \\
\hline Amplio (C) & 95,6 & \\
\hline
\end{tabular}

a: Prueba de Mann Whitney.

Tabla 4. Comparación de la fuerza de tracción con la medida del conducto

\begin{tabular}{|c|c|c|}
\hline Grupo & Mediana fuerza de tracción & $\mathrm{P}^{\mathrm{a}}$ \\
\hline Angosto (A) & 63,5 & \\
\hline Amplio (B) & 101,0 & 0,003 \\
\hline Amplio (C) & 148.0 & \\
\hline
\end{tabular}

De acuerdo con la prueba ANOVA el valor de $\mathrm{p}$ fue igual a 0,064 lo que sugiere aceptar la hipótesis nula que indica que las medianas de la resistencia al desalojo de los tres grupos son iguales como se observa en la Tabla 5. Mediante esta prueba se encontró una diferencia estadísticamente significativa al comparar la fuerza de tracción en los tres grupos $(p=0,005)$; como se había mencionado, los valores más altos se obtuvieron en el grupo $C$ en el que se realizo un refuerzo radicular con resina y tal vez esta fue la razón por la que hubo una mayor fuerza de tracción para producir el desalojo tal como se reporta en la Tabla 6.

Tabla 5. Comparación de la resistencia al desalojo entre los grupos A, B y C.

\begin{tabular}{cccc}
\hline Grupo & Estadístico & Valor & $\mathbf{P}^{\mathbf{a}}$ \\
\hline \multirow{2}{*}{ A } & Promedio & 79,2 & \\
& Mediana & 43,0 & \\
\multirow{2}{*}{ B } & Promedio & 99.9 & 0,064 \\
& Mediana & 52.6 & \\
C & Promedio & 159.3 & \\
& Mediana & 95,6 & \\
\hline
\end{tabular}

a: Prueba ANOVA

Tabla 6. Comparación de la fuerza de traccion en los grupos A, B y C.

\begin{tabular}{cccc}
\hline Grupo & Estadístico & Valor & $\mathbf{P}^{\mathbf{a}}$ \\
\hline \multirow{2}{*}{ A } & Promedio & 103,3 & \\
& Mediana & 63,5 & 0,005 \\
\multirow{2}{*}{ B } & Promedio & 136,1 & \\
& Mediana & 101,0 & \\
C & Promedio & 212,8 & 148,0 \\
\hline
\end{tabular}

a: Prueba ANOVA. 


\section{Modo de falla}

Al momento de aplicar fuerzas de tracción en la máquina universal de ensayos, las muestras presentaron mayores fallas adhesivas en la interfase dentina-material restaurador, ya que al acondicionar la

Tabla 7. Ubicación de las fallas adhesivas superficie dentinal hay formación de una capa híbrida que proporciona el recurso más importante para obtener valores altos de adhesión y buen sellado de la interfase material restaurador-dentina. La ubicación de las fallas se observa en la Tabla 7.

\begin{tabular}{lccc}
\hline \multicolumn{1}{c}{ Tipo de falla } & Grupo A & Grupo B & Grupo C \\
\hline Falla Poste - Cemento & 8 dientes & 4 dientes & 11 dientes \\
Falla Dentina - Cemento & 10 dientes & 13 dientes \\
Falla Dentina - Resina & & & 9 dientes \\
\hline
\end{tabular}

\section{DISCUSIÓN}

Los resultados de este estudio mostraron que se encontró una diferencia estadísticamente significativa en las fuerzas de tracción que se necesitaron para producir la dislocación del poste de fibra de vidrio desde el conducto radicular y la mayor fuerza fue aplicada al grupo con refuerzo radicular. Jara y colaboradores midieron y compararon las diferentes fuerzas de tracción necesarias para la dislocación de pernos de fibra de vidrio desde el conducto radicular cementados con Panavia F 2.0, Unicem, Fuji plus y Variolink y observaron resultados similares a los hallados en esta investigación. ${ }^{16}$

Saupe y colaboradores compararon la resistencia a la fractura de los dientes tratados endodónticamente con paredes radiculares debilitadas y la instalación de un poste adosado al socavado, en los cuales se reforzaron las paredes radiculares con resinas compuestas y el poste se cementó posteriormente; demostraron que las raíces comprometidas y reforzadas con resina compuesta, ofrecieron un 50\% más de resistencia a la fractura, que las raíces con un poste adosado al socavado (sin refuerzo). ${ }^{12}$ En el presente estudio, la técnica del refuerzo radicular aplicada a los dientes pertenecientes al Grupo $C$ resultó ser la más efectiva para el fortalecimiento de estructura radicular debilitada.

Por su parte, Garita y Rodríguez encontraron que el cemento de resina auto grabable presentó mayor adhesión a la superficie del poste que a la dentina del canal radicular; el cemento Relyx Unicem en cuanto a valores absolutos de retención, presentó en promedio el valor más alto al ser comparado con el Variolink II y el Fuji Plus. ${ }^{11}$ Igualmente en este estudio, el cemento de resina auto grabable (Relyx U200, 3M ESPE ${ }^{\circledR}$ ) presentó mayor adherencia al poste en fibra de vidrio y no a la superficie dentinaria ya que el cemento resinoso tiene buena adherencia a la fibra de vidrio, este presenta una óptima combinación de alta fuerza de flexión, alta rigidez, baja inicial rigidez y adecuada radiopacidad.
La presencia de la capa híbrida en la interfase dentina-material restaurador, aumenta la habilidad de estos sistemas de adhesión de unirse al sustrato dentinario para sellar la superficie de la dentina y eliminar casi por completo el flujo de fluidos en la interfase y disminuir la sensibilidad posoperatoria propia de estos procedimientos. ${ }^{17}$ Se evidenció que los postes en fibra de vidrio presentaron mayor adhesión con el cemento resinoso (Relyx U200, $3 \mathrm{M} \mathrm{ESPE}^{\circledR}$ ) al producir mayor retención con la resina compuesta y mostrar el principal desprendimiento con la dentina y no con el poste.

Se aplicaron fuerzas de tracción a las muestras cementadas con la técnica convencional para los grupos A y B que presentaron menor resistencia al desalojo, en tanto que al grupo $C$ se le aplicó la técnica de refuerzo radicular que fue la más efectiva en el fortalecimiento de la estructura radicular debilitada. Al comparar las dos técnicas (convencional y refuerzo radicular) se evidenció que existe menor adhesión entre la interfase dentina-cemento y dentina-resina en los tres grupos, mientras que el comportamiento presentado entre poste-cemento indicó que los postes presentaron mayor adhesión con el material restaurador. Los dientes que pertenecían al grupo $C$ a los que se les aplicó el refuerzo radicular en conductos debilitados demostraron mayor resistencia a la fuerza de tracción posiblemente, debido a que la resistencia de la estructura dental se incrementó cuando el canal radicular es reforzado con resina compuesta.

La técnica que presentó mayor resistencia al desalojo fue la técnica de refuerzo radicular. Sin embargo, la técnica convencional es efectiva en el caso de rehabilitar dientes con conductos radiculares angostos y en el caso de conductos radiculares amplios es conveniente utilizar la técnica de refuerzo radicular porque demostró que este refuerzo le genera mayor retención al poste.

Es importante mencionar que el estudio sólo evaluó la fuerza a la tracción de dos técnicas de cementa- 
ción de postes prefabricados con los materiales antes mencionados; las fuerzas oclusales y funcionales que se presentan en boca son de tipo compuesto por lo que se puede concluir que un poste de fibra de vidrio cementado no puede fallar solo por someterse a fuerzas de tracción, sino por fuerzas de compresión o cizallamiento que se generan de manera conjunta.

\section{BIBLIOGRAFÍA}

1. Forni DP. Resistencia a las fuerzas de tracción de cemento de resina cemntos de vidrio ionómero modificado con resina. Estudio In vitro. Universidad de Talca; 2005. URL disponible en: http://dspace.utalca.cl/bitstream/1950/2130/1/forni_fuentes.pdf

2. Mahn G. Evaluación morfológica de la interacción del adhesivo Adper Prompt-L-Pop con la dentina del conducto radicular [Trabajo de Grado]. Santiago de Chile: Universidad de Chile; 2005.

3. Vallejo M, Maya C, Erazo N. Resistencia a la fractura de dientes con debilitamiento radicular. CES Odontol 2011; 24: $59-69$.

4. Chávez-Valdivia ND. Resistencia a la fractura de piezas dentales restauradas con anclajes de fibra carbono y colados. Estudio In vitro [Trabajo de Grado]. Lima: Universidad Nacional Mayor de San Marcos; 2002.

5. Fakiha Z, Al-Aujan A, Al-Shamrani S. Retention of cast posts cemented with zinc phosphate cement using different cementing techniques. J Prosthodont 2001;10: $37-41$.

6. Ojeda F, Puente F, Goldaracena MP, Montero VM. Estudio In vitro de resistencia a la fractura de dientes tratados con endodoncia y restaurados con dos sistemas de postes. ADM 2011; LXVIII: 290 - 297.

7. Palomino BC. Evaluación de la resistencia a las fuerzas de tracción de coronas completas cementadas con cemento de ionómero vítreo, ionómero vítreo reforzado con resina y cemento resinoso dual sobre preparaciones dentarias no pulidas [Trabajo de Grado]. Lima: Universidad Peruana Cayetano Heredia; 2006.

8. Mallat E. Ionómeros de Vidrio (I) Geodental [en línea] 2002. [Fecha de acceso: febrero de 2013]. URL disponible en: http://clinicamallat.com/05_formacion/art_cien/miscelanea/mi02.pdf

9. D'Arcangelo C, D'Amario MD, Vadini M, Zazzeroni S, De Angelius FD, Caputi S. An evaluation of luting agent application technique effect on fibre post retention. J Dent 2008; 36: 235 - 240.

10. Garita A, Rodríguez C. Comparación In vitro de la fuerza de retención en endopostes de fibra de vidrio prefabricados entre los cementos de resina autograbable, cemento de resina convecional y cemento de ionómero de vidrio modificado con resina. Revista IDental 2008, 1: 25 - 35.

11. García A. Estudio In vitro comparativo de la fuerza de adhesión de un ionómero y dos resinas utilizadas para adherir brackets [Trabajo de Grado]. Lima: Universidad Nacional San Marcos; 2002.

12. Saupe WA, Gluskin AH, Radke RA Jr. A comparative study of fracture resistance between morphologic dowel and cores and resin-reinforced Dowel System in the intraradicular restoration of structurally compromised root. Quintessence Int 1996; 27: 483 - 491.
13. Lawley R, Chindler W, Walker Kolodrubetz D. Evaluation of ultrasonically placed MTA and fracture resistance with intracanal composite resin in a model of apexification. J Endod 2004; 30: 167 - 172.

14. Mazzitelli C. Evaluación de la unión entre cementos resinosos-adhesivos y la dentina [Trabajo de Grado]. Granada: Universidad de Granada; 2008.

15. Rodríguez JB. Adhesión de selladores endodónticos a centina radicular [Trabajo de Grado]. Granada: Universidad de Granada; 2007.

16. Jara P, Martínez A, Correa G, Catalán A. Estudio In vitro de la resistencia a la tracción de postes de fibra de vidrio cementados con cuatro agentes cementantes. Avances en Odontoestomatología 2010; 26: 255 - 262.

17. Medina D, Kaplan A, Avalos M. Mecanismos de falla en postes de fibra de vidrio. CONAMET-SAM [en línea] 2008. [Fecha de acceso: marzo de 2013]. URL disponible en: http://www.materiales-sam.org.ar/sitio/biblioteca/ CONAMET-SAM2008/pdfs/g6.pdf

\section{Correos electrónicos de los autores:}

Juliana Elisa Celis Corzo: julianita.celis@hotmail.com Adriana Cáceres Marulanda:nanita_0806@hotmail.com Juan Carlos Cabrera Rojas: juanc_1970@yahoo.com.mx Jorge Guillermo Díaz Rodríguez: jorgegdiaz@gmail.com 